\title{
A Study on the Virtual Reality Interface Integrated Logistics Support System
}

\author{
Hanggeun Shim ${ }^{1}$, Jaekyung Yang ${ }^{2}$ and Myoungjin Choi $^{3 *}$ \\ ${ }^{1}$ Hanwha Defense R\&D Center 10, Yuseong-daerol 1366beon-fil, \\ Yuseong-gu, Daejeon, 34101, Republic of Korea \\ ${ }^{2}$ Department of Industrial \& Information Systems Engineering, Chonbuk National \\ University, 567 Baekje-daero, Deokkjin-gu, Jeonju-si, Jeollabuk-do, 54896, \\ Republic of Korea \\ ${ }^{3}$ Department of Defense Science \& Technology, Howon University. 64 \\ Howondae3-gil, Impi-myon, Gunsan-si, Jeollabud-do, 54058, Republic of Korea \\ ${ }^{1}$ hanggeun@hanwha.com, ${ }^{2} j k y a n g @ j b n u . a c . k r,{ }^{3 *}$ officesky@howon.ac.kr
}

\begin{abstract}
Integrated logistics support should be developed in parallel when conducting research and development of weapon systems. However, the opportunity for concurrent development of technical manuals for maintenance and supply support is limited. Therefore, we propose a virtual reality maintenance system to address these problems. The proposed system uses XML format, automatically produces maintenance sequences from $3 D$ design data, validates maintenance tasks using virtual reality technology, automatically develops logistics support analysis, and produces technical manuals. The system provides a virtual environment interface that displays and controls the high precision model data, so the user experiences a close to real environment.
\end{abstract}

Keywords: Interactive Electronic Technical Manual, Logistics Support Analysis, NDBG Algorithm, Sequence, Task Virtual Validation, Virtual Reality

\section{Introduction}

To develop integrated logistics support(ILS) for weapon systems, the equipment and prototype configuration are required for logistics support analysis(LSA) and generating the technical manual (TM). However, the final product is often somewhat different from initial prototypes due to frequent design changes during development, resulting in excess work and cost. Virtual reality (VR) techniques using design data will help reduce costs and to minimize inefficient work to automatically general the technical manual and provide an educational or training tool. This paper describes the framework of a VR ILS, initially called Virtual Reality Maintenance System (VRMS), and discusses its necessity.

\section{Virtual Reality Maintenance System Necessity}

\subsection{Background and Purpose}

Technical manuals (TMs) include maintenance task guidelines, illustrated drawings, support equipment manuals, and software manual(s). They provide guidance for mechanics and technicians to maintain and manage weapon system safely and efficiently. The maintenance TM describes maintenance sequence(s) to complete tasks safely and efficiently, necessary spare parts, special or common tools, and personnel requirements.

Received (October 9, 2017), Review Result (December 19, 2017), Accepted (January 24, 2018)

${ }^{*}$ Corresponding Author 
Logistic support analysis (LSA) databases include all aspects of LSA documentation and management, such as LSA control number (LCN) assignment, function analysis (item identification data), failure modes and effects analysis (FMEA), criticality analysis (CA), reliability centered maintenance analysis (RCMA), economic analysis, level of repair analysis (LORA), reliability availability maintainability (RAM) matrix, maintenance task analysis, maintenance engineering analysis, input/output data formats, etc.

Maintenance without TMs is not practical, since modern weapon systems are complex equipment, with complicated electronic and electrical circuit, hydraulic, and mechanical configurations. Weapon systems development represent significant capital investment, and require precise TMs to enable integrated logistics support (ILS) tasks to be developed in parallel with equipment design. The TM must include all maintenance, supply, and required equipment and actions based on comprehensive procedures and engineering statistical analysis provide effective allocation of repairs and maintenance expense and effectiveness. Thus, TMs are absolutely essential to improve equipment reliability and availability, and minimize repair and maintenance expenses.

Recently, the Agency for Defense Development (MADC-501-030073, 2003; MADC415-040591, 2004; MADC-415-040592, 2004) introduced the concept of interactive electronic technical manual (IETM) development to automatically generate basic maintenance procedures and TMs in conjunction with those generated from current ILS procedures. The IETM system uses LSA analysis data, manual illustrations or drawings, and enables tracking management with efficient corrections promotion. The concept contributes strongly to improved operability of main equipment in two ways: improved productivity of TM development by integrating editing and feedback for illustrated drawings, documents, charts, and data from current practices, and taking advantage of electronic production. Computer aided management utilizing internet technology and client-server environments allow a universal electronic technology bridging the manual system, but separate from the web based environment by developing user friendly systems and function controls to replace and add functionality.

Badler (2000) developed an automated maintenance instruction (AMI) to reduce the effort and cost to develop maintenance manuals, and a service manual generation (SMG) system to produce and validate the manuals. These systems electronically integrated the production of TMs, and provided a new paradigm providing more accurate, enhanced results with minimum cost and effort.

There is a growing need for a system that secures accurate weapon systems data such as shape and combination information from 3D CAD data, that is consistently maintained and renewed through ILS procedures, and substitutes current labor intensive, intuitive methods that rely heavily on expert experience. Similarly, maintenance task verification with desired design specifications and feedback should take advantage of a virtual environment to minimize constraints of requiring actual systems and reduce the discontinuity between development, manufacturing, and maintenance. Therefore, automatic production and verification of maintenance procedures using virtual rather than actual systems minimizes effort and cost to develop more precise TMs

\subsection{Current Status and Problems}

Accurate maintenance task analysis for main weapon systems enables reliable and precise TM development. However, existing manual processes have no option but to trial manufactured goods for maintenance task analysis as shown in Figure 1. This labor intensive process must be performed by maintenance task analysts utilizing their intuition and experience. Managing time and costs to verify weapon systems is difficult, causing time constraints, and leading to delayed manual production. Consequently, the final manual may not match the required analytic results or current figuration, and will be less precise than optimal. There is also significant time and cost to acquire weapon systems and develop maintenance education and follow up support, because maintenance tasks 
must be conducted with actual weapons system in an operational state. Therefore, verifying maintenance tasks and developing maintenance education in a virtual environment will reduce time and costs, and also produce more precise TMs and educational tools.

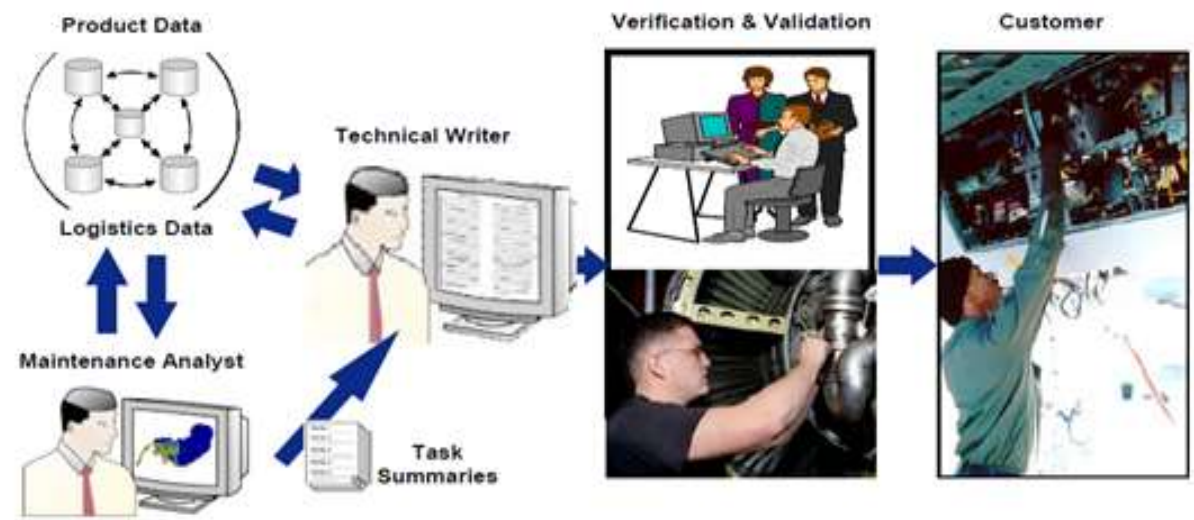

Figure 1. Technical Manual Development Procedure

Maintenance task verification and developing logistics support is indispensable before manufacturing trial parts throughout weapon systems development and concurrent engineering methods. Verification and utilizing maintenance training equipment using by virtual reality (VR) systems is very effective. Virtual Reality technology includes head mounted displays (HMDs), data gloves, motion trackers, haptic devices, etc. It is also essential to identify design errors when verifying maintenance sequences by interaction between the user and objects using VR systems.

Current weapon systems development provides maintenance task analysis, including maintenance tasks and supply information, collected in the TM. The task analysis system provides data integrity through interaction between various information sources. There is a growing need to introduce VR for verification from design to operation, because the VR environment preserves coherence, minimizes incoherent information, and promotes efficiency. The technology is close to being ready for practical use.

Assembly and disassembly based on VR instructions, verifying maintenance procedures, and examining related issues requires related data, tools, supporting equipment, supplies, etc., and must eventually extend to mechanical training because this comprehensive and systematic development approach method innovatively improves time and cost for weapon systems development.

Therefore, this paper introduces the concept of an ILS information system that generates maintenance procedures from design data, validates the generated procedures using VR, studies maintenance task utilization, provides information for previously developed LSA systems, and generates TMs and IETMs to produce a VR maintenance training system

\section{Current Systems}

\subsection{Service Manual Generation}

\subsubsection{Basic Concept and Definition}

Service manual generation (SMG) is a development project of automated maintenance manuals, associated with the Air Force Dual Science and Technology Office, General Electronic Global Research Technology Office, and Lockheed Martin. SMG introduces a 
new concept of the maintenance manual development framework. This reduces development cost and time by automated search and combination of maintenance data.

SMG consist of three main technical factors.

- Service Sequence (SS): provides the disassembly sequence.

- Task Generation (TG): creates a draft Manual draft using SS.

- Virtual Validation (VV): provides immersion in a VR environment

The SMG project has shown that that increased integration of TM development provided superior information with lower cost in shorter time.

\subsubsection{Main Technical Factors}

\subsubsection{Service Sequence}

The SMG system encodes the design shape in three dimensions, and uses a visualization tool interface to interact with data and SS. The SS generates the disassembling sequence actions to perform specific maintenance task, as shown in Figure 2. The non-directional blocking graph (NDBG) technique was introduced to find the fastest disassembly path for assembled products by searching for obstacles in the directions disassembled parts need to follow. The results from SS, disassembly path sequences, and related images and animations are saved in extensible markup language (XML) format, which accommodates disassembled drawing information as well as maintenance sequence details.

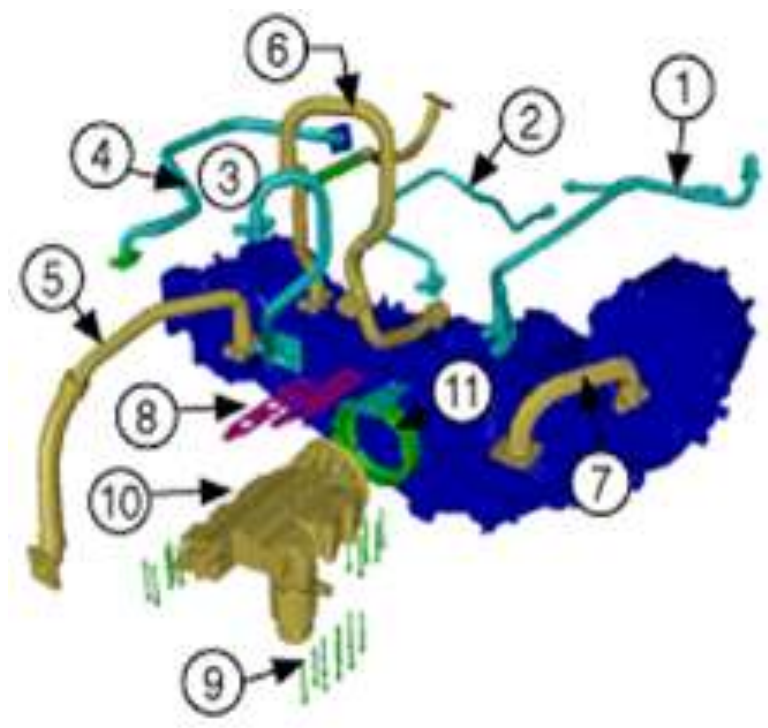

Figure 2. Solid Model Service Sequence Graphic

\subsubsection{Task Generation}

Creation of the maintenance statement of work requires an equipment geometric model, including maintenance task steps, safety warnings, required tools, repair parts, and written explanations. The TG module takes the service sequences and related images as input and outputs a logical form in XML format, following the algorithm shown in Figure 3. 


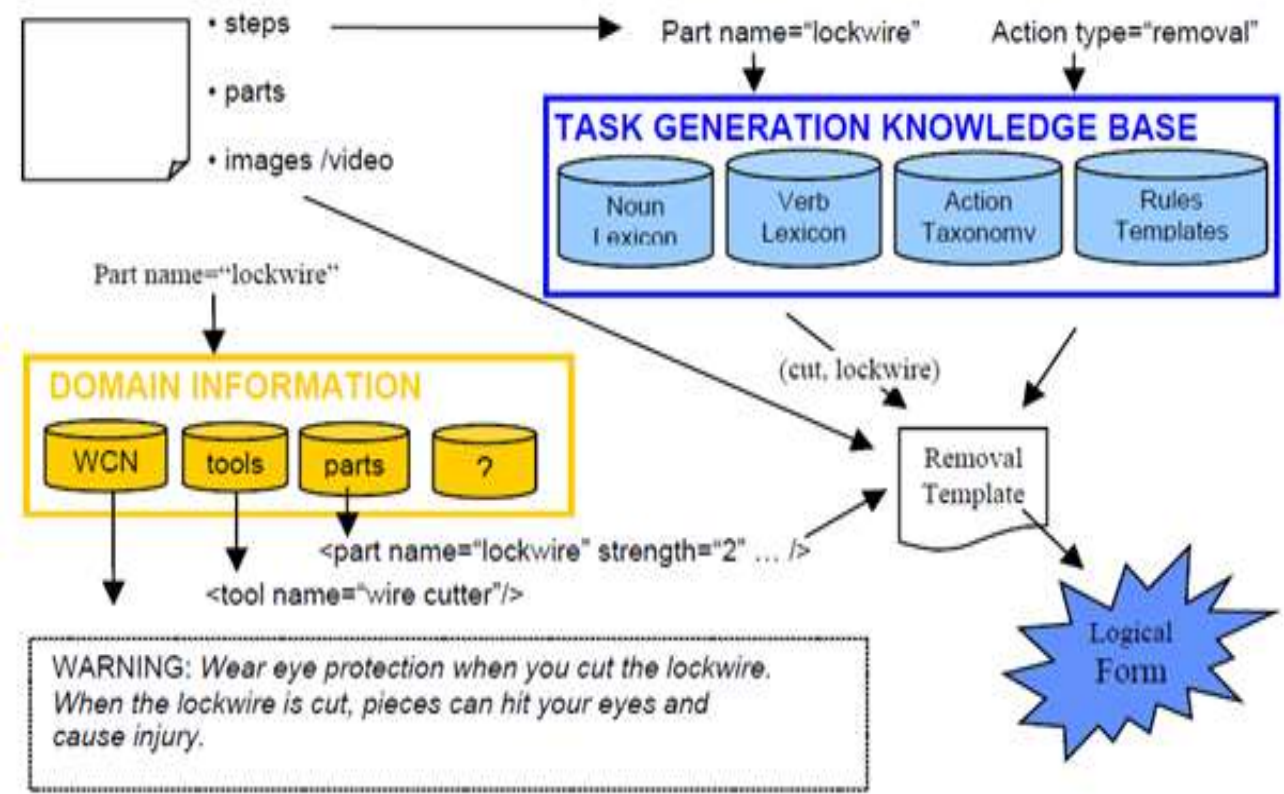

Figure 3. Logical Form Generation

\subsubsection{Virtual Validation}

Combining manual production and engineering design data in SMG requires a unique and innovative solution to allow maintenance procedure validation. Design data from CAD systems was employed in a haptic virtual environment, through a PC and integrated various VR devices, including HMD, data glove, body tracker, haptic devices, etc.

VV consists of two parts: service sequence (first validation) examines the proposed procedure; and then a second validation of the TG output that an analyst can effectively perform the task, using an assortment of texts and images, as shown in Figure 4.

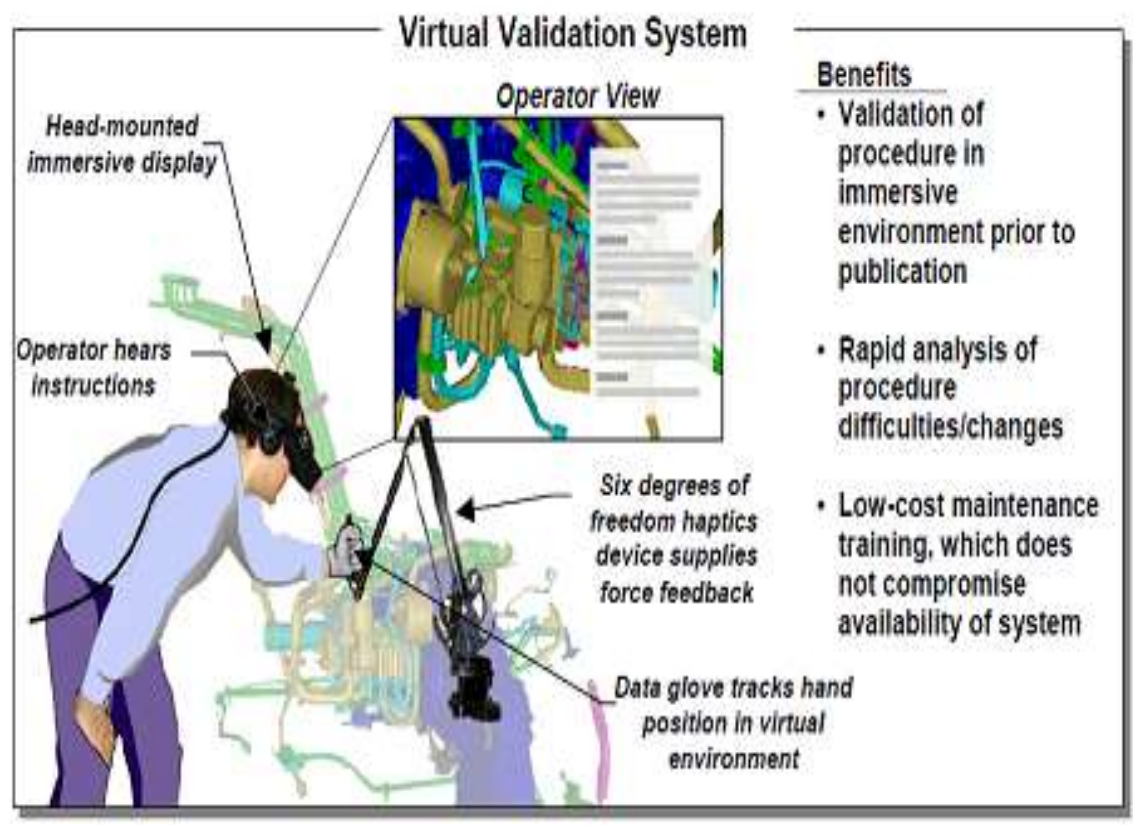

Figure 4. Virtual Environment 
A maintenance display was provided to users visually using an HMD. Head movement was tracked by magnetic tracker, and the virtual screen was dynamically updated.

Volpe (2002) showed how users can control virtual equipment or tools using a data gloves and haptic devices. Users recognize forces and torques through the haptic device when they touch virtual objects.

As shown in Figure 5 is a flow chart of the maintenance manual development using SMG

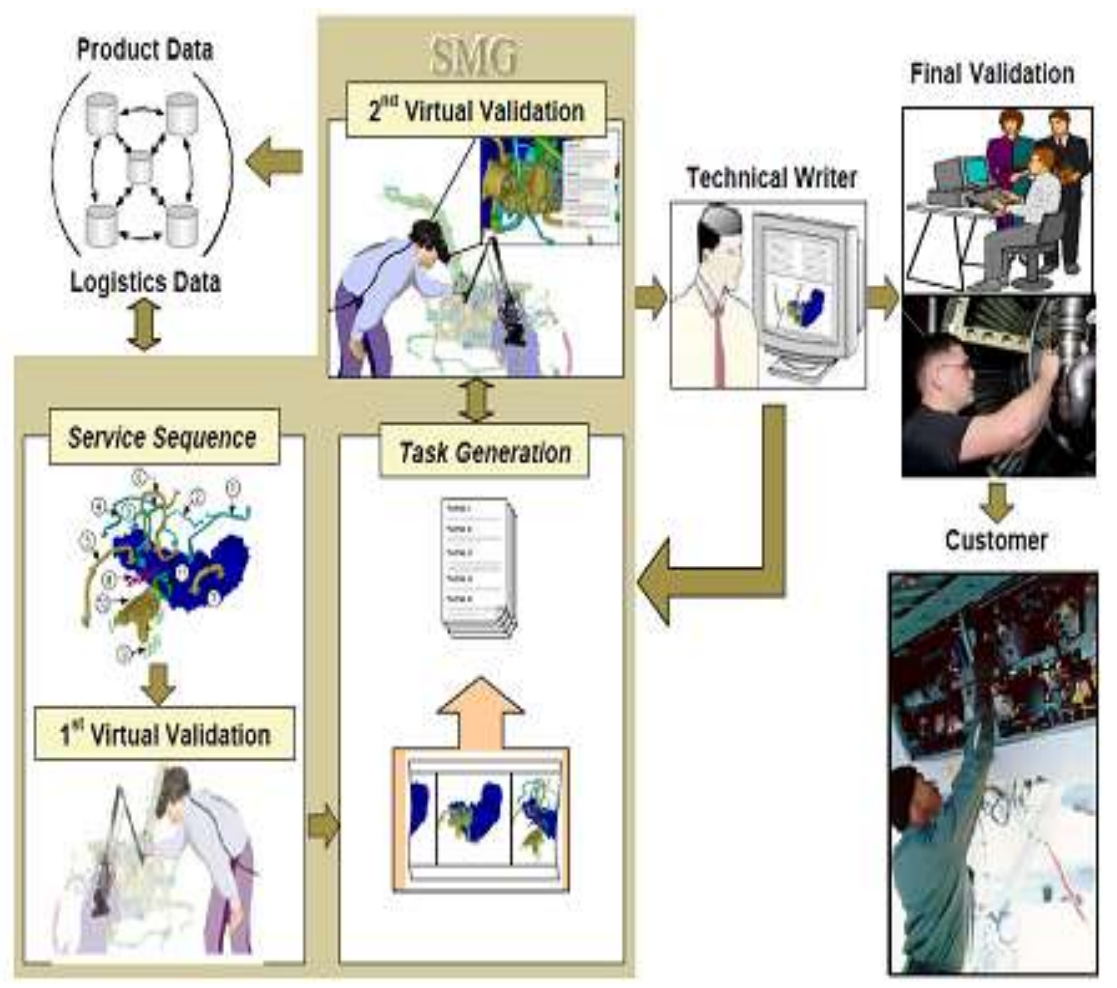

Figure 5. Maintenance Manual Development using SMG

\subsection{Technical Manual Generation}

\subsubsection{Concept and Definition}

The Technical Manual Generation (TMG) system is

An integrated system that is feasible of authoring/printing of paper manuals and IETM with suitability of logistics support and Technical Manual operation system by authoring/managing through manual generator, illustrated drawing writer, and constructing illustrated drawing systems using data from CAD, input data from LSA program and consists of four main systems:

- technical manual management (TMM),

- technical manual authoring (TMA),

- illustrated drawing management (IDM), and

- illustrated drawing authoring (IDA)

This study unified LSA and manual development concepts by integrating LSA and TM databases for TM development. This reduced cost and manpower, and provided a new generation of ILS infrastructure. 


\subsubsection{System and Main Functions}

\subsubsection{Technical Manual Management}

TMM sets the environment and manages TM production with four main functions:

- import LSA database(s),

- manual registration and production,

- manage national defense standards (NDS), and

- manage contractor file integration.

\subsubsection{Technical Manual Authoring}

Wampler (2002) developed a technical manual authoring (TMA) system that automatically records LSA data for a registered TM, and edits data to complete TMs. The TMA incorporates four main functions:

- conjunction with the LSA databases,

- conjunction with the IDA system,

- Integrate the MIL-PRF-87269A database for manual authoring and editing, and

- automatically generate NDS forms based on LSA.

\subsubsection{Illustrated Drawing Authoring and Management}

Illustrated drawing authoring and management (IDA/IDM) acquires material and shape information from 3D illustrated drawings. The system includes 5 main functions:

- provide the unique illustrated drawing number,

- conjunction with drawing number and information, manage Authoring Drawing File,

- integrate the supply catalog and illustrated drawings (Hot Spot),

- 3D shape Explode and generate 2D contours, and

- Provide a shape spot and view relation log.

\section{Virtual Reality Integrated Logistics Support Development Program}

\subsection{System Concept and Definition}

As discussed above, there is a growing need for development and effective system integration based on existing systems. The current study proposes a virtual reality maintenance system (VRMS) that automatically provides validation for analyzed or generated maintenance tasks using VR technology; integrates automatic TM generation, minimizing validation using the actual object; provides LSA analysis from design data, reducing repetitive revisions when changing design; and maintains the consistency of design, LSA, and TM information using XML based data management. The VRMS system can also be used for training and simulation rather than requiring extra equipment production these purposes, and allows test and evaluation of maintainability at design level.

Using the proposed VRMS, maintenance analysts can validate maintenance procedures early in the design process using a virtual environment. The system allows cost effective identification and modification of high risk design flaws, and integrates maintainability 
with concurrent engineering. Many tasks can be automated by integrating current LSA and TM generating systems.

The VRMS comprises three main components: sequence generation (SG), task generation and validation (VV), and operator interface (Interface).

\subsection{Sequence Generation Module}

The SG module generates maintenance procedures. Preferentially 3D design data is imported into the SG module to analyze maintenance procedures and tasks. Common 3D CAD data formats can be easily modified for use in the module.

Importing the 3D design data generates disassembly sequences using the sequence algorithm in the maintenance procedure generation system using NDBG, which defines blocking relations between parts in respective directions when considering removal ensure a valid disassembly sequence. This makes it easier and faster to decide if a specific part shape is removable or not. Generated maintenance sequences are saved in XML format and exported to the VV module. The first virtual validation of the maintenance sequence considers the logicality of the maintenance sequence, then the maintenance procedure is exported to the TG module.

\subsection{TG Module}

Not only shape data, but also information such as description, warnings and cautions, required tools, and repair parts for the maintenance sequence are required to generate maintenance instructions. The TG module generates the logical form of the SG module in XML format.

The generated maintenance sequence from the SG module receives saved XML data and selects an appropriate logical template format. The module then generates and runs SQL statements in the appropriate maintenance sequence, searching for information, such as maintenance instructions, warnings or cautions, tool and repair parts, then integrates and documents them. Saved information is exported to the VV module, and maintenance instructions and related information are validated through a second virtual validation process. Subsequently, all relevant information is exported to the LSA database.

\subsection{Virtual Validation Module}

The VV module automatically generates maintenance procedures from design data, and validates generated procedures. The VV module comprises two major sections.

- First validation: validates the SG module generated maintenance procedure.

- Second validation: validates the accuracy of generated maintenance instructions and images from the TG module, and checks that mechanics could effectively complete maintenance tasks.

The VV module validates maintenance tasks using VR devices operated by the maintenance analyst using XML data in 3D. The analyst wears an HMD, providing an immersion type VR environment. The virtual screen point of view changes dynamically according head movement sensed by magnetic tracker. Interactions between virtual parts are enabled using a data glove and haptic device. When the user touches a virtual object, force and torque are applied through the haptic device. Thus, the immersion VR allows maintenance analysts to validate maintenance procedures before producing a TM. The module provides much faster analysis for difficult or change-required design parts, and can be utilized as a cost efficient training tool without requiring actual equipment. 


\subsection{Interface Module}

The Interface module exports validated data to the LSA TM generation database for LSA and TM development for each LCN section. Concurrently, information regarding repair parts, special tools, etc. can be exported to the supply manual. When the analysis is completed, the exported information maybe utilized to generate a suitable TM.

\subsection{System Environment}

Figure 6 shows the proposed VRMS operation.

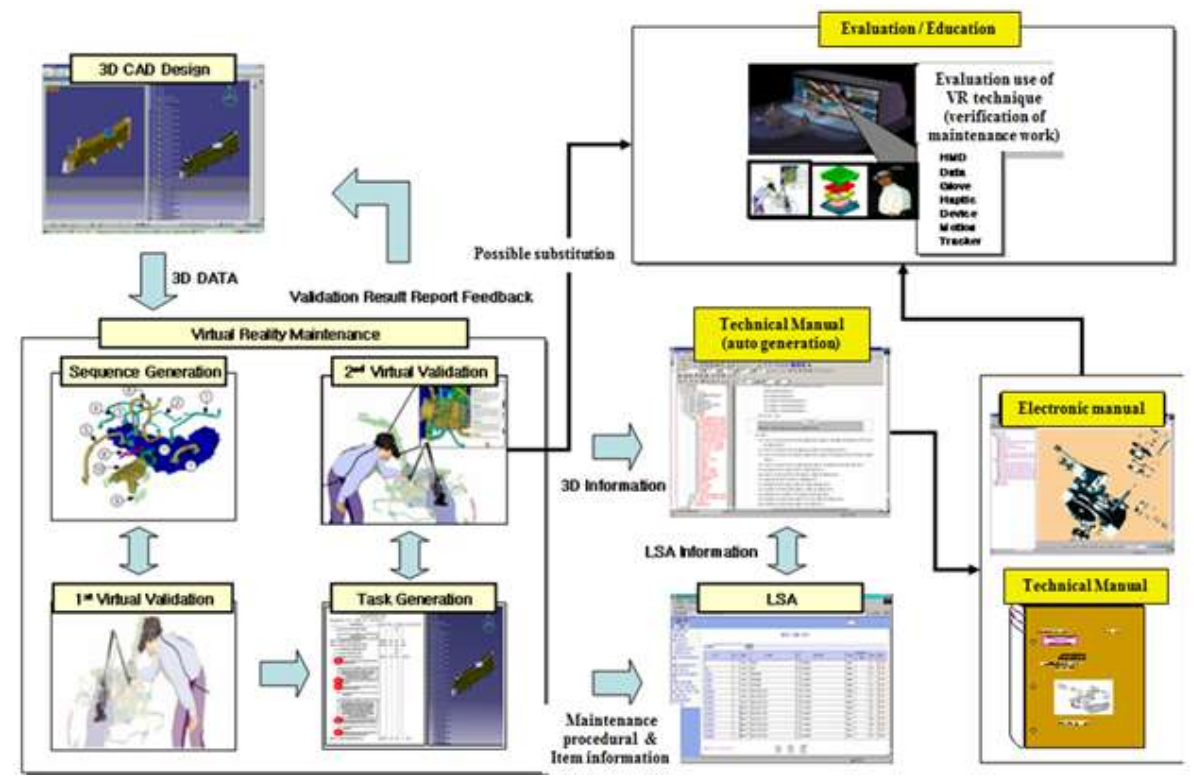

Figure 6. Virtual Reality Maintenance

\section{Conclusions}

The proposed VRMS automatically generates maintenance procedures from 3D design data, validates maintenance tasks using VR technology to confirm maintainability, and utilizes automatically generated data for LSA and TM generation. Particular features of the proposed system are described as follows.

- Automatic maintenance task generation and analysis from 3D design data. This replaces current labor intensive, intuitive methods that depend solely on maintenance analyst experience.

- Task validation uses virtual reality rather than actual equipment for generated maintenance tasks to allow maintenance analysts to validate maintainability in 3D.

- Generated and analyzed maintenance tasks, whether automatically or by maintenance analysts using the VR system, are linked to LSA and automatic TM generation. This enables construction of infrastructure for ILS tasks, such as maintenance task analysis, LSA, and TM generation.

The proposed Virtual Reality Maintenance System will provide a strong tool for computerization of ILS element data and VR based training system.

\section{Acknowledgements}

This research was supported by research funds from the Internal Research Support Programs of Howon University in 2017. 


\section{References}

[1] N. Badler, C. Erignac, P. Vincent, E. Sanchez, J. Wampler and J. Ianni, "Design Concepts for Automating Maintenance Instructions", Air Force Research Laboratory Technical Report (AFRL-HEWP-TR-2000-0088), (2000).

[2] MADC-501-030073, IOTA-XP Basic Design II (DB Design and Program), Research Report MADC501-030073, Agency for Defense Development, South Korea, (2003).

[3] MADC-415-040591, Technical Manual Generation Software Specific Design Description (SDD), Research Report MADC-415-040591, Agency for Defense Development, South Korea, (2004).

[4] MADC-415-040592, Technical Manual Generation Software Database (OBDD), Research Report MADC-415-040592. Agency for Defense Development, South Korea, (2004).

[5] C. R. Volpe and R. Blue, "Virtual Haptic Validation for Service Manual Generation", GE Global Research Center, (2002).

[6] J. Wampler, R. Blue, C. R. Volpe and P. Rondot, "Service Manual Generation: An Automated Approach to Maintenance Manual Development", Proceedings of the 16th Human Factors in Aviation Maintenance Symposium, (2002).

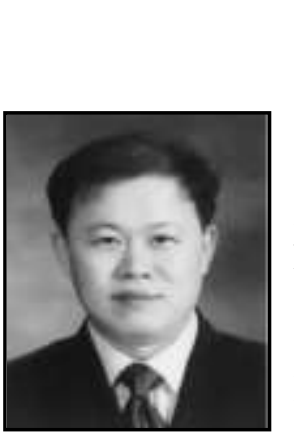

\section{Authors}

Hanggeun Shim, he is currently a group leader in the ILS group of Defense R\&D Center at Hanwha Corporation in Republic of Korea. He received his BS degree in Dept. of Mechanical Engineering at Inha University in 1989, and MS degree in Dept. of Defense of Military Science (Logistics acquisition) at Chungnam National University in 2014 respectively. His current research interests include Defense logistics engineering, virtual reality training system and weapon system.

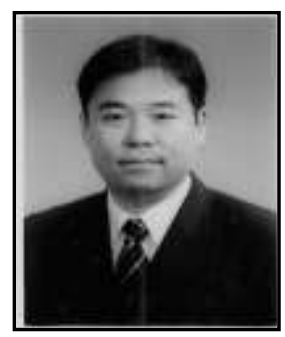

Jaekyung Yang, he is currently an professor in the Department of Industrial and Information Systems Engineering at Chonbuk Nationl University in Republic of Korea. He received his BS degree in Industrial Engineering from Hanyang University, MS degree in Industrial Engineering from Korea Advanced Institute of Science and Technology (KAIST), and Ph.D. degree in Industrial and Manufacturing Systems Engineering from Iowa State University in 1992, 1994, and 2003 respectively. Before he joined Chonbuk National University, he worked as a senior researcher at ETRI and a senior consultant at Samsung SDS. His primary research interests are in data mining, intelligent systems, logistics and supply chain management.

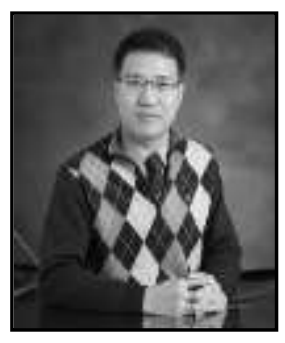

Myoungiin Choi, he is currently an associate professor in the Dept. of Defense Science \& Technology at Howon University in Republic of Korea. He received his BS degree in Dept. of Industrial Engineering at Soongsil University, MS degree in Dept. of Defense \& Information management at Korea National Defense University, and Ph.D. degree in Industrial and Information Systems Engineering at Chonbuk National University in 1999, 2009 and 2016 respectively. His current research interests include data mining, national defense information system and weapon system. 